\title{
A Picture Is Worth a Thousand Tags: Automatic Web Based Image Tag Expansion
}

\author{
Andrew Gilbert and Richard Bowden \\ Centre for Vision, Speech and Signal Processing, \\ University of Surrey, Guildford, GU2 7XH, UK \\ $\{$ a.gilbert, r. bowden\}@surrey.ac.uk
}

\begin{abstract}
We present an approach to automatically expand the annotation of images using the internet as an additional information source. The novelty of the work is in the expansion of image tags by automatically introducing new unseen complex linguistic labels which are collected unsupervised from associated webpages. Taking a small subset of existing image tags, a web based search retrieves additional textual information. Both a textual bag of words model and a visual bag of words model are combined and symbolised for data mining. Association rule mining is then used to identify rules which relate words to visual contents. Unseen images that fit these rules are re-tagged. This approach allows a large number of additional annotations to be added to unseen images, on average 12.8 new tags per image, with an $87.2 \%$ true positive rate. Results are shown on two datasets including a new 2800 image annotation dataset of landmarks, the results include pictures of buildings being tagged with the architect, the year of construction and even events that have taken place there. This widens the tag annotation impact and their use in retrieval. This dataset is made available along with tags and the 1970 webpages and additional images which form the information corpus. In addition, results for a common state-of-the-art dataset MIRFlickr25000 are presented for comparison of the learning framework against previous works.
\end{abstract}

\section{Introduction}

The quantity of images captured and stored by users grows at an ever increasing rate, fuelled by on-line social networking sites like facebook and Flickr. Searching such content is limited by the textual tags people give them. Often the images are tagged by only a few high level keywords, however, we propose an approach to automatically enrich images with further textual annotations describing objects, locations, the objects history, and actions occurring within the image. Rather than using a large training set of groundtruthed labelled images, we use text sources harvested from the internet to automatically provide the additional tags. There have been many examples in the literature of image annotation and tag expansion however they often concentrate on large generic datasets [1 3]. These approaches are good at providing high level categorisation of images, for 
example, the results for the image in Figure 1, would be Road, Building, Sky and City. These category level tags are acceptable for generic tagging of images but do not provide very informative tags for searching an image database. We concentrate on introducing new tags not specified by the user but mined from general webpages. This is the main novelty of the approach. The goal is to tag such an image with tags which might include Big Ben, London, UK, London bus, Charles Barry and 1858. The latter two being the architect who designed Big Ben and 1858 the year its construction was completed. Both of which would make excellent search terms for this image. To expand the tag annotations manually would be time consuming, therefore we propose to novelly use content from textual web pages also automatically harvested from the internet. These web pages may contain additional images and text which can be used to expand the annotation of the input image. In the next section we look at related work.

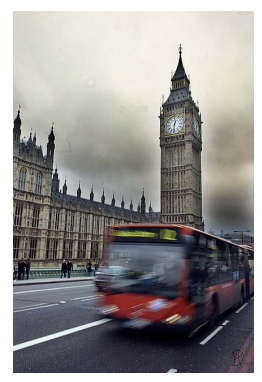

Fig. 1. Big Ben in London in the UK

Section 3 explains the basic methodology behind the tag expansion procedure. Following a brief overview we explain how we represent images as symbol sets, how APriori mining is used to find the relationship between visual features and textual tags and how information from webpages is then used to retag images. Section 4 presents tag expansion results on a new dataset and the approach is further validated or the simpler task of assigning known tags to images on a benchmark dataset before conclusions are drawn.

\section{Related Work}

This work has much in common with the work by Farhadi [4] in that they wished to move from basic level categories towards attributes to describe objects. For example, instead of the category cat, they would describe the cat as having a tail, and ears and legs. They did this through learning and inferring attributes to improve the overall recognition of the objects. A number of works [5, 1] have approached large scale labelling of images but being developed by large scale corporations they rely heavily on distributed processing. Many approaches have been proposed to solve annotation including generative, discriminative, and nearest neighbour approaches. Generative topic models annotate images as samples 
from a specific mixture of topics, where each topic is a distribution over image features and annotation tags. Examples of topic models include Dirichlet allocation [6] and hierarchical Dirichlet process models [7]. However these can be limited as they learn a joint distribution over a large set of features and images, which would need to be scaled to deal with large modern web based datasets. Discriminative models have also been proposed for image annotation including SVMs [8] and by learning distance functions [9]. These methods learn a separate classifier for each keyword and are therefore more suited to a low number of keywords. Nearest-neighbour-based methods [10 12] have become more attractive recently since the amount of training data is rapidly increasing. TagProp, the approach by Guillaumin [10] for auto image annotation uses an effective nearest neighbourhood model, by taking a weighted combination of keyword absence and presence among its neighbours. Makadia [11] poses image annotation as a retrieval problem, nearest neighbours are determined by the average of several feature distances. Near duplicate image identification can also be used [12]. The use of hierarchical structures including trees [13] and DAGs [14] are increasingly used to model the dependencies between the text tags, as the dataset sizes and complexity increases. However, nearest neighbour approaches can only tag new images with tags which are represented in the training image corpus. This is the key difference to our approach, rather than annotation an image with existing text tags, our goal is to introduce new and expand tags to the corpus using the internet as an additional source of information.

\section{Tag Expansion Methodology}

Figure 2 shows an overview of the approach we propose. In order to expand the tag annotation of an image, the existing text tags from the image are used as internet searches. The resulting top $n$ pages are harvested for images and text. For each page, and each image, the corresponding text from the web page and image features are extracted into an generic container called an image descriptor and added to the corpus of tagged images. Association rule datamining is then used to identify links between visual features and text labels. APriori data mining [15] finds rules based on combinations of image features that relate to a specific and distinctive piece of text (a keyword). By comparing the image features from the original image, to the mined rules, the image can be annotated with the additional text. Effectively we use existing tags to query the internet for content, then learn the relationship between image features and semantic textual labels through data mining. These rules are then used to re-tag the images and for a dataset of 2800 images of 14 landmarks, the approach is demonstrated to provide on average 12.8 additional text tags per image, in a completely automatic fashion. We further validate the approach on the MIR-Flickr-250000 dataset by tagging unseen images with comparable performance to the state-of-the-art. 


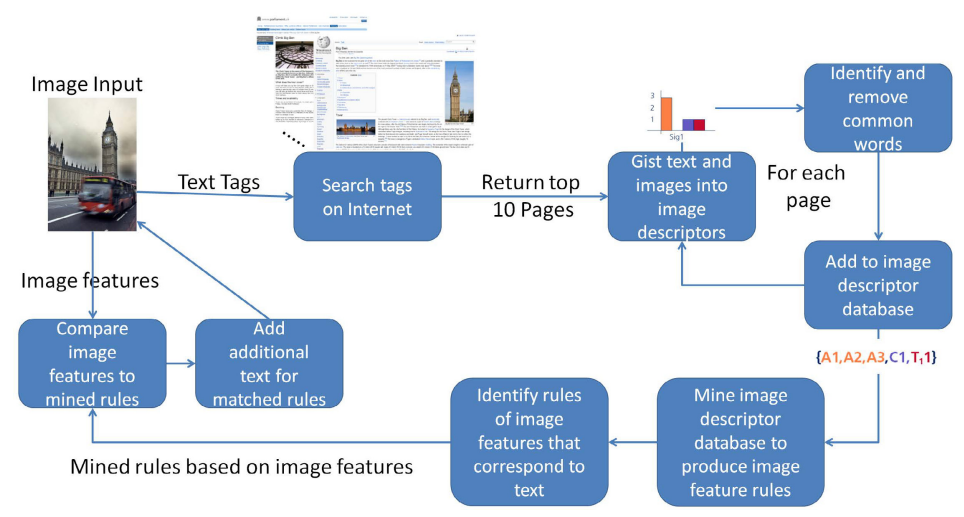

Fig. 2. An Overview of the approach

\subsection{Image Descriptor}

The image descriptor is used to summarise information on both the images and the text tag information in a form that allows data mining to determine how the image features relate to the text tags. We adapt the container used by Gilbert and Bowden [16] who proposed an image signature, which is a frequency histogram based representation of a image feature that is converted to a symbolised one dimensional set. We take this idea of symbolisation, which is ideal for the data mining and use it to build feature descriptors that describe both the visual features (visual Bag of Words) and text tags (traditional textual Bag of Words) for each image. For each image a Bag of Words model is extracted based on SIFT features [17]. Before constructing the codebook, the SIFT features are reduced in dimensionality using the feature dimensionally reduction technique of Cai, Mikolajczyk and Matas [18]. The approach uses Linear Discriminant Projections (LDP) for reducing dimensionality and improving discrimination of local image descriptors. LDP can reduce the dimensionality of SIFT without compromising the performance and outperforms the commonly used PCA. This makes it a useful tool for this computationally demanding large-scale image recognition problem. The LDP projects each descriptor to 30 dimensions which improves efficiency while maintaining recognition accuracy. The dimensionally reduced features are computed over all the images and the features are quantised using standard kmeans clustering to form a codebook of 4000 bins. A bag of words histogram is formed for each image using a soft assignment approach to form a frequency based histogram of the feature descriptors. Textual words already form a natural discrete codebook and the textual bag of words and the visual bag of words are simply concatenated. The number of possible text tags, can be high and sparsely populated, however, this is of little concern as the symbolism of the image descriptor ignores the unused bins. The weighted histogram is converted into a set of symbols where each symbol corresponds to a word in the histogram and the number of occurrences of each symbol corresponds 


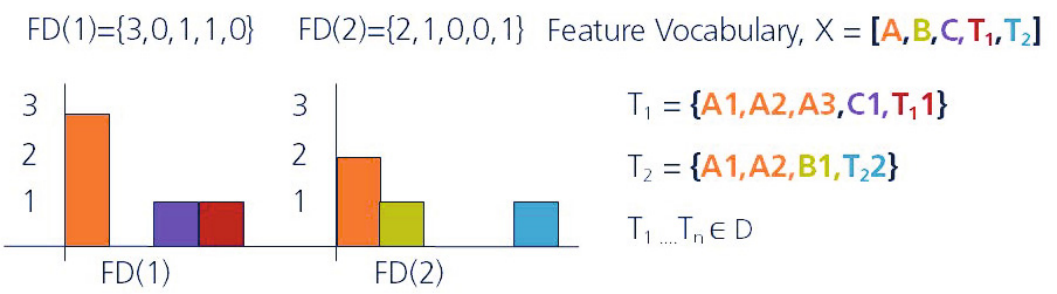

Fig. 3. The symbolisation of the image signature

to its frequency. Figure 3 gives an overview of this process. Given an input image that has a feature descriptor $\mathrm{FD}(1)$ consisting of a histogram of 3 visual words, and 2 text tags and a frequency response of $\{3,0,1,1,0\}$. It is converted into a 1D set of symbols using the frequency of each feature to duplicate each feature in turn into a set of symbols. In the example above, the vocabulary becomes $\left\{A 1, A 2, A 3, C 1, T_{1} 1\right\}$. For another example where the image descriptor is $\{2,1,0,0,1\}$ the symbolised form becomes $\left\{A 1, A 2, B 1, T_{2} 1\right\}$. The repetition of symbols will be used in computing the support during the mining process.

\subsection{APriori Data Mining}

We wish to identify co-occurring words within the data. Specifically, groups of visual words which commonly occur with textual words or tags. To do this we use a variation of association rule mining called the APriori algorithm [19, 15]. An association rule, is a relationship of the form $\{A, B\} \Rightarrow C$, where $A$, $B$, and $C$ are sets of items. The frequency of an item set is related to the support and confidence for an association rule. If $A$ is an item set and $D$ the bag of all transactions under consideration, then the support of the item set $A$ is the number of transactions in $D$ that contain $A$. The support for an item set measures the statistical significance i.e. the probability that a Transaction, $T$ contains the item set. For $A$, this is calculated as the size of the set of all $T$, such that $T$ is an element of $D$ and $A$ is a subset of $T$, normalised by the size of $D$. The support of the rule $A \Rightarrow B$ is therefore

$$
\sup (A \Rightarrow B)=\frac{|\{T \mid T \in D,(A \cup B) \subseteq T\}|}{|D|}
$$

and measures the statistical significance of the rule. The goal of frequent item set mining is to find all item sets (that is, all subsets of the item base) that occur in the given bag of transactions with at least a user-specified minimum support. When mining for association rules, it is no good just finding the most frequent as these will be frequent in all text tag classes, therefore discriminative rules are required. To measure the discriminability of association rules, Agrawal et al [15], introduced the confidence of a rule. The confidence of an association rule is the support of the set of all items that appear in the rule (the support 
Table 1. Most frequent text tags before and after common text removal

\begin{tabular}{|c||c|c|}
\hline & $\begin{array}{c}\text { Most Frequent } \\
\text { Before }\end{array}$ & $\begin{array}{c}\text { Most Frequent } \\
\text { After }\end{array}$ \\
\hline 1 & a & UK \\
\hline 2 & the & London \\
\hline 3 & that & palace \\
\hline 4 & we & Westminster \\
\hline 5 & I & Big Ben \\
\hline 6 & of & Spain \\
\hline 7 & at & Thames \\
\hline 8 & in & Gaudi \\
\hline 9 & is & Canary Wharf \\
\hline 10 & with & river \\
\hline
\end{tabular}

of $S=\{A, B\})$ divided by the support of the antecedent of the rule (here $A$ ). Therefore the confidence is,

$$
\operatorname{conf}(A \Rightarrow B)=\frac{\sup (A \cup B)}{\sup (A)}
$$

More intuitively, the confidence of a rule is the number of cases in which the rule is correct relative to the number of cases in which it is applicable. It states this percentage relative to the number of cases in which the antecedent holds, since these are the cases in which the rule makes a prediction that can be true or false. If the antecedent does not hold, then the rule does not make any prediction, so these cases are excluded. Rules are reported as association rules if their confidence reaches or exceeds a minimum confidence. Therefore the support for the rule is the probability of the joint occurrence of $A$ and $B$ i.e. $P(A, B)$ while confidence is the conditional probability $P(B \mid A)$. For each element $T$ of $D$, we are interested in finding the sets of image features $M_{i}(\alpha)$, that also contain the specific text tag, $\alpha$ such that $\operatorname{con} f(M(\alpha) \Rightarrow \alpha)>C$ and $\sup (M(\alpha) \Rightarrow \alpha)>S$. For all experiments in this manuscript, $C=80 \%$ and $S=1 \%$, this ensures only rules that contain image features that do not often occur with other text elements are returned. This means the features are discriminative with respect to the other text tags. A very low support ensures that nearly all rules are examined.

\subsection{Automatic Tag Expansion}

Given an image taken from Flickr or facebook it may already contain tags that have been manually appended to the image by the original user, the aim of this work is to take these category tags and automatically expand them to provide a far richer description of the image. Novelly, no further user intervention is required as the approach is able to automatically determine the correct tags that relate to the image. In order to perform the tag expansion, the text tags that are embedded in the image are used as the input to a web search, and 
text and images from the top $n$ returned web pages are retrieved and converted into new image descriptors. The aim is to use the images from the web pages in conjunction with the text to learn the features that occur when certain text occurs. Obviously, any image descriptor that comes from an image and tag will have extremely sparse entries in the textual bag of words whereas an image from a webpage that has all the text associated with the image will result in a heavily populated histogram. However, there is a large amount of common text within the web pages that has little to do with the semantic meaning. This will include words like $a$, the, that which etc. These are definite and indefinite articles, and non descriptive with respect to tag expansion and must be identified and removed. A simple approach would be to filter using a list of such articles. However a more context aware approach is to use a first stage of APriori mining to filter the data. This has the advantage of being fast due to the efficiency of the mining algorithm. In order to achieve this, the text from a small subset of 10 negative web pages is symbolised into image descriptors, and a 0 is appended to the image descriptor to indicate they are negative examples. The text from the other image descriptors is appended with a 1 to indicate they are the positive examples, and both are combined into a transaction database. The mining is run over the database, and $\alpha$ is set to 1 . The returned rules will consist of the text items that won't occur on the negative data, the non common words can then be used to remove common text tags from the image descriptors. Once the common words are removed, the mining can be reused to identify the image features that consistently occur with specific text tags. The aim being that if these image features are found on the original image, the additional text tags can be appended to it. In order to achieve this, the image descriptors formed of images and non common text from the internet are fed to the APriori algorithm again. The data mining uses high confidence and low support to determine rules made up of compounds of image features that occur only for a specific text tag $\alpha$. Then, given an unseen or new image, if the compound of image features for a specific rule occur within an image, the image is appended with the text tag $\alpha$. Figure 4 gives examples of sets of visual features that correspond to a text tag. The matching of the features with the yellow circle correspond to the tag Thames, the name of the river while the features represented by the black square correspond to the rule 1858, this was the year that Big Ben was built. It is likely that some of the tags used as the input to the web search are unwanted such as camera models or adjectives like busy or blue. In these cases, the rules of compound image features returned by mining are unlikely to match with the original images and therefore the words are not added as tags.

\section{Results}

In order to demonstrate the power of the approach, we present results on two datasets. Firstly we demonstrate the applicability of automatic tag expansion on a custom dataset called Image Tag which we also make available to the wider community. This is a small dataset of around 2800 images extracted from Flickr 

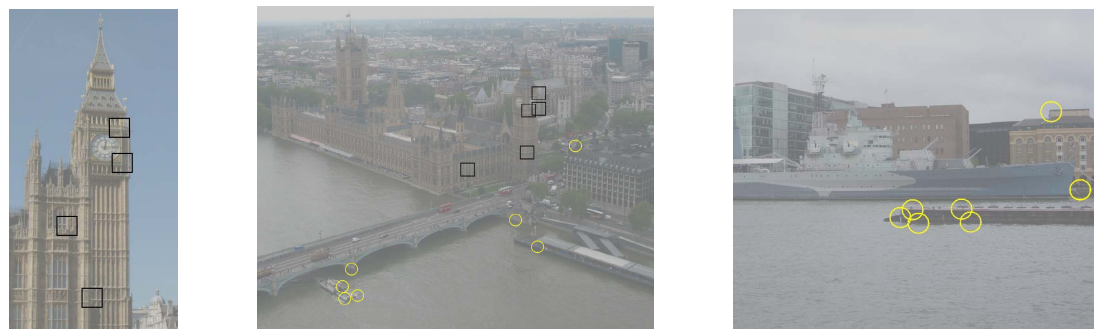

Fig. 4. Examples of the image feature compounds rules

along with 1970 related web pages which allows the linguistic web content to be used to both tag new images and expand existing tags in the dataset. The 2800 images are evenly spread across 14 classes of landmarks from both London and Barcelona. The associated web pages were the top $n=10$ returned by Google for each of the image tags used in the Flickr dataset. This dataset is used to demonstrate the ability of the approach to automatically assign new tags to images based on additional harvested linguistic information. In the second set of tests, we use the ImageCLEF2010 dataset to evaluate the approach at automatic photograph annotation. While the linguistic content of this dataset is limited to just tags, it provides a comparison to state of the art approaches in the ImageCLEF2010 Photo Annotation competition.

\subsection{ImageTag}

Image Tag consists of 2800 images and associated meta-data from the internet image site Flickr. The dataset consists of 200 images and meta data for each of the 14 classes of tourist sites in London and Barcelona consisting of: Big Ben, Buckingham Palace, Canada Square, Casa Mila, HMS Belfast, London Bus, Sagrada Familia, St Pancras, St Paul's, Torre Agbar, Tower Bridge, Tower of London, Wembley, Westminster Abbey. Figure 5 gives examples of the images and some of the tags harvested. There are 197 unique tags across the 2800 images. Around $20 \%$ of the images contain no text tags, and can contain foreign languages, and spelling mistakes. For each of the tags, the top $n=10$ web pages returned from Google were also collected and their content in terms of both text and images harvested. This results in 1970 web pages giving a corpus of around 39,348 words. The dataset is split into training and test partitions, the training consists of $10 \%$ of the images randomly selected from each class (20 per class), making a training set of 320 images and text tags. The test data is formed from the remaining data which is 180 examples from each of the 14 classes, making 2280 images. The dataset is available from http://www.andrewjohngilbert.co.uk/data.html 


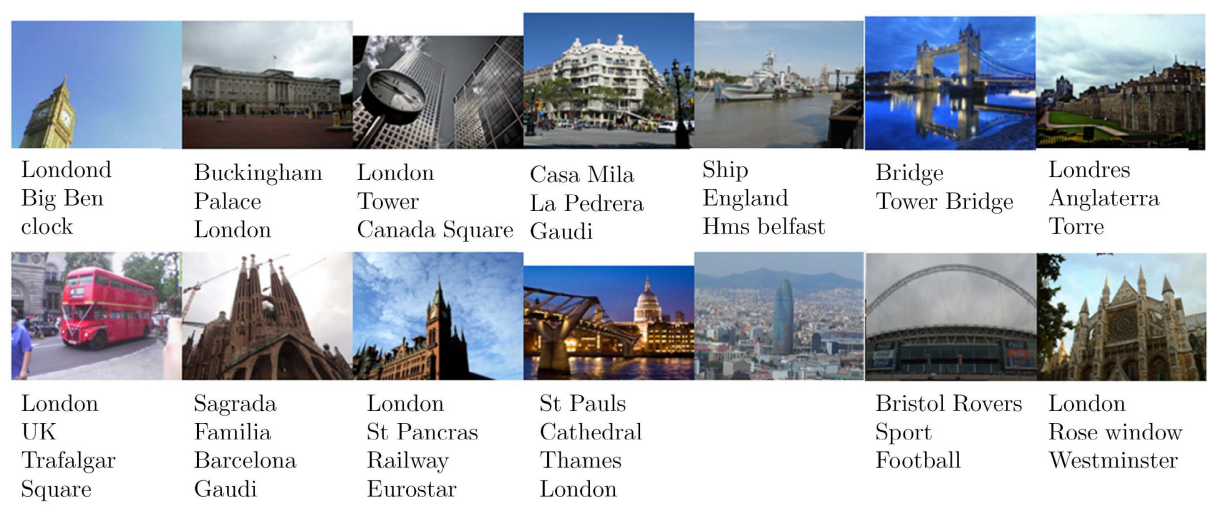

Fig. 5. Example Images and Tags from the ImageTag Dataset

\subsection{Extracting Candidate Tags}

Initially there are 39,348 candidate text tags returned from the various web pages. The first step is to remove common words that have no discriminatory meaning. APriori data mining is applied over the image descriptors, and rules $M_{i}(\alpha)$ found for each text tag $\alpha$. The text tag instances are reduced to 18,390 . Table 1 shows the effect of the data mining in removing the common or article text from the websites. The left column shows the 10 most frequent text words from the websites before and after mining.

\subsection{Recognition}

Mining now extracts the rules $M_{i}(\alpha)$ for each potential text tag $\alpha$ and for any empty sets, the candidate tag is removed. To expand tags for an unseen image $q$, it is analysed in a similar fashion to training. The dimensionally reduced SIFT features are detected and quantised into the bag of words frequency histogram $F D(q)$. This is symbolised into a query image descriptor $T(q)$. This query descriptor is then compared to the mined rules and if the rule $M(\alpha) \subseteq T(q)$, then the score for the text tag, $\alpha$ is incremented by the confidence of the rule $\operatorname{con} f(M(\alpha) \Rightarrow \alpha)$. Once all rules have been checked, any text tag that exceeds a threshold is assigned to the image. The effect of this threshold is shown in Figure 8 .

\subsection{ImageTag Results}

To show the results on the ImageTag dataset, we show the outcome of tag expansion. The unseen images are automatically annotated with tags from the mined image feature rules. Some of these tags will be tags that exist in the images groundtruth, the category level tags such as building or London, and these are used to form a precision and recall graph. This graph is shown in Figure 6. The 


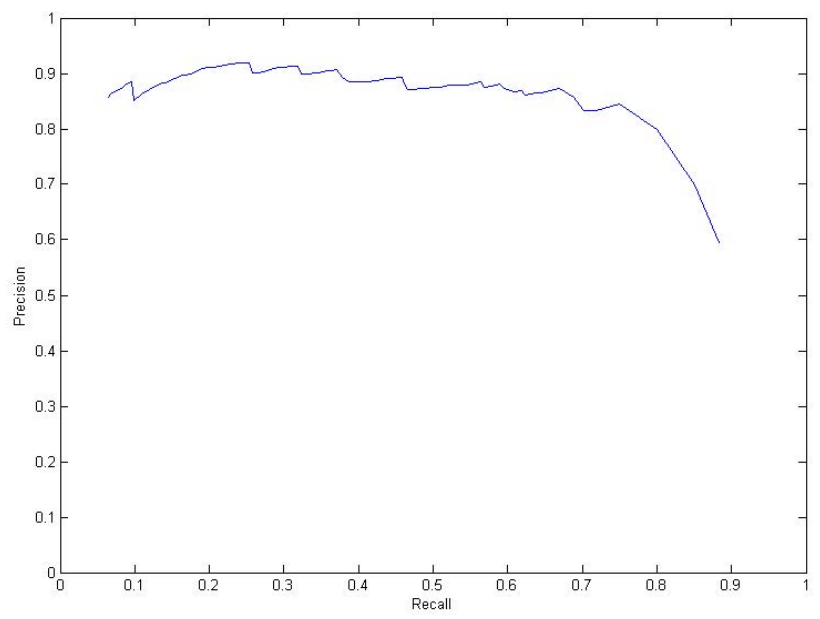

Fig. 6. The Precision recall of our approach on the ImageTag Dataset

graph shows the approach has a relatively stable precision. The graph validates the ability of the approach to simply reproduce the tags provided by the user. However, the approach is able to extend the tags for the images, and this is our main novel contribution. On average, each image is annotated with 12.8 additional complex linguistic text tags. These text tags are able to provide far more information than a standard user would. This is because the images are being tagged with the keywords harvested from detailed internet web pages. The computation of the process to expand the text tags takes 3 hours in a single core machine,although parallelisation would reduce the time considerably. Figure 7 shows images with their additional annotated text tags, it can be seen that quite subtle information has been extracted from the web pages. For example, the picture of Big Ben has additional information about the architect and the year of construction, while for Torre Agbar, additional information about the number of floors and its official opening date have been added. There are also false positive tags due to incorrect matching of the image features, the picture of Westminster Abbey contains some of the same feature rules as Big Ben, and is therefore incorrectly labelled. Figure 8 shows the ROC curve for the ImageTag Dataset for the different classes. An overall True Positive rate of $87.2 \%$ with a $12.4 \%$ false positive rate is achieved when adding additional tags to the unseen images. The ROC curve shows there is a variation between the different classes, the class London bus is the worst performing, this is probably due to the wide intra class variation possible for the class. While the classes Canada Square and Torre Agbar perform well, this is possible due to the very distinctive appearance of the images, and also because there is a smaller range of web pages available to describe the images. 


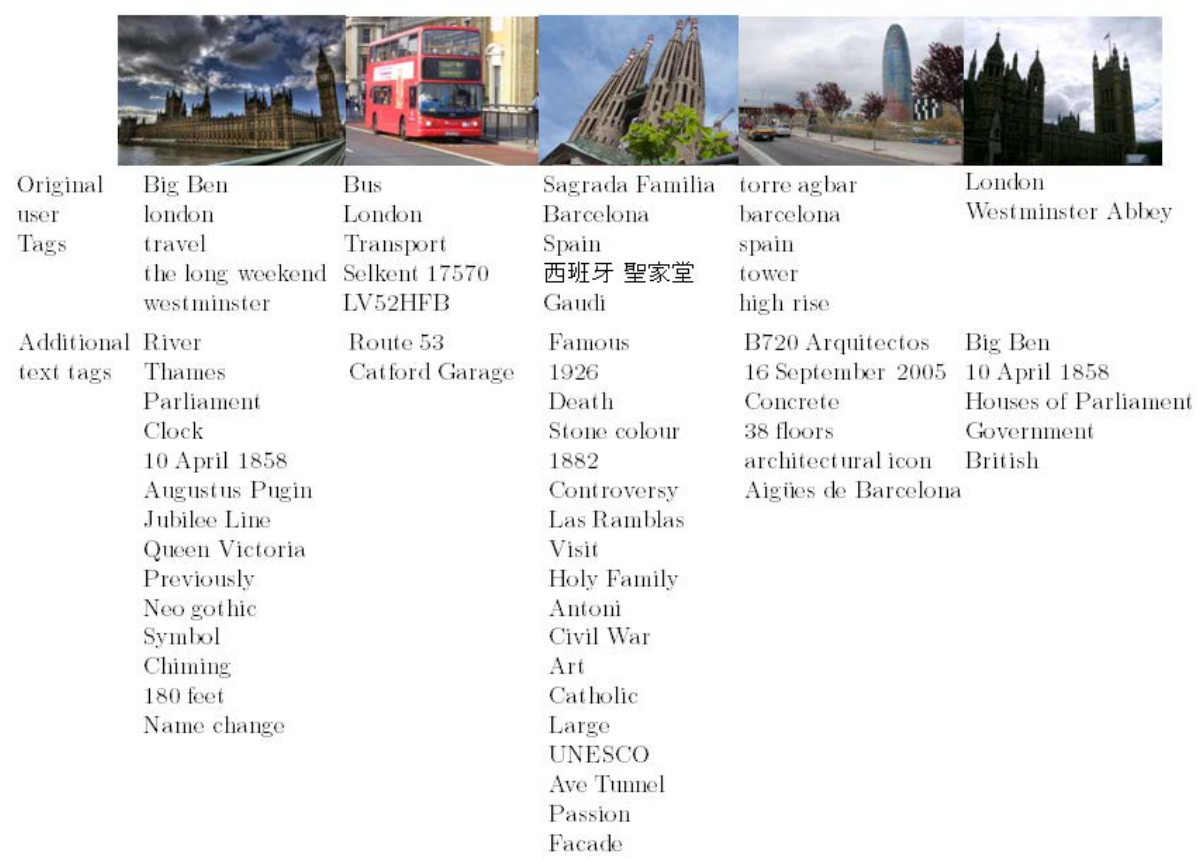

Fig. 7. Examples of Text Tag expansion on the ImageTag Dataset

\subsection{ImageCLEF2010 Tests}

In order to compare with other results we perform the automatic image annotation with text tags on the MIR-Flickr-25000 dataset [20]. This dataset is used to demonstrate that we have not over fitted to a specific problem but actually solved a more general one. It is a standard image annotation dataset comprising of 25000 images also from Flickr. Each image also contains text tags users have appended to describe the image, on average each image has 8.94 tags and there are 1386 tags that occur in at least 20 images. Some examples of the popular tags are sky, water, portrait, night, sun, red, this indicates that the tags are quite different to the ones from the ImageTag dataset. Some of the tags also refer to more abstract concepts love, travel or to adjectives old, cute, vintage and red. In order to compensate for the differing tag styles and to show the generalisation of the image descriptor, in addition to the SIFT and tag features, a colour quantisation histogram (6 bins per channel) and a GIST scene descriptor [21] is concatenated to the image descriptor. The visual photo annotation task from ImageCLEF2010 was used to test the approach, the training set consisted of 8,000 Flickr images, and the test set of 10,000. The image descriptors from the training image set were data mined with respect to the text tags, $\alpha$. The resultant discriminative mined rules, $M(\alpha)$ were rules made up of compounds of image features that correspond to a specific text tag. In order to annotate the unseen test images, the 


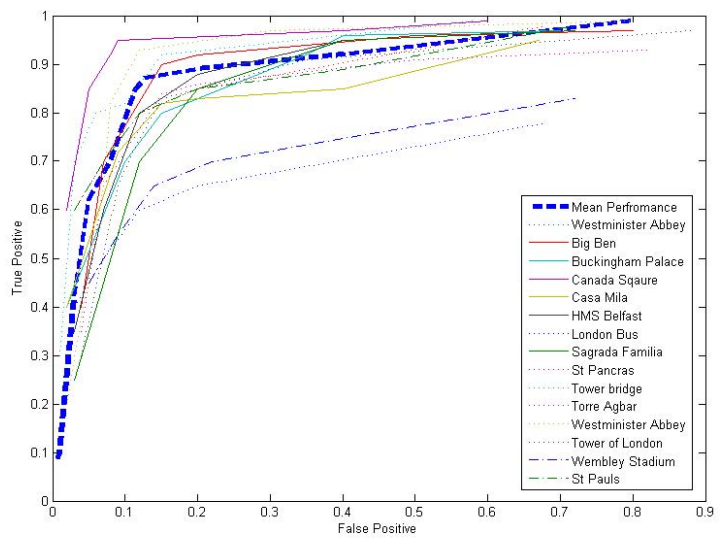

Fig. 8. ROC Curve of Text Tag expansion on the ImageTag Dataset

image descriptor for each image was taken in turn and compared to the mined rules. If the query transaction has a mined association rule then the score for the text tag, $\alpha$ would be incremented by an amount equal to the confidence of the association rule. The scores for all the tags are accumulated and a threshold used to assign the text tag to the image. Table 4.5 shows a selection of results, together with the proposed approach for both SIFT features alone and also for SIFT/GIST/RGB colour histograms. The approach takes around 1 day for the complete experimental process to complete on a single core desktop machine with $32 \mathrm{~GB}$ of RAM. The results on the ImageCLEF2010 task are impressive

Table 2. Results for ImageCLEF2010 photo annotation

\begin{tabular}{|c||c|}
\hline Approach & MAP \\
\hline ISIS [22] & 0.407 \\
XRCE [22] & 0.390 \\
VisualSynset [1] & 0.364 \\
TagProp [10] & 0.360 \\
HHI [22] & 0.350 \\
IJS [22] & 0.334 \\
BPACAD [22] & 0.283 \\
Romania [22] & 0.259 \\
Proposed Approach (SIFT) & 0.281 \\
Proposed Approach (SIFT+GIST+RGB) & 0.362 \\
\hline
\end{tabular}

considering the approach is designed primarily for query expansion rather than tag annotation. Especially when once considers that the SIFT codebook used in both tests was calculated on the ImageTag dataset and further improvements would be expected if it was tailored to the ImageCLEF2010 dataset. This is 
confirmed by the per class performance. Out of the 93 concepts/classes, our approach beats the state-of-the-art on 27 of those concepts. These 27 concepts are based around external landmarks and architecture which is what our SIFT codebook has been optimised for. While the SIFT descriptors could have optimised over the ImageCLEF2010 dataset to ensure we had the top result, we are more interested in recalculating a general codebook which would give a more balanced representation over both the datasets. Demonstrating that the approach performs equally well at the simpler task of tagging images with known labels.

\section{Conclusion}

We have introduced a new approach to expand the text tag annotation of an image by introducing new tags not present in the image corpus. To achieve this we combine automatic searching of the internet using existing tags with association rule mining to find rules which link visual bag of word features to textual bag of word descriptors. We report results on two complex image datasets gaining a high mean average precision on the challenging ImageCLEF2010 challenge, while using fewer feature types than alternative approaches and despite a non optimal image codebook. This validates the feature mining approach. However, our main research question was how to expand image tags using information harvested from the internet. To achieve this we employ the same feature learning approach on a new ImageTag Dataset and demonstrate the expansion of the tag annotation by an average 12.8 tags per image which are quantitatively evaluated to provide an $87.2 \%$ true positive rate. We further qualitatively evaluate the results, demonstrating that the additional tags can capture detailed knowledge about the image content, rather than simple categories. Future work will extend the approach to include more features and larger datasets as the generic image descriptor and efficient mining approach provides a scalable solution to both dataset size and the number of features used.

\section{References}

1. Tsai, D., Jing, Y., Liu, Y., Rowley, H., Ioffe, S.M., Rehg, J.: Large-scale image annotation using visual synset. In: Proc. of IEEE International Conference on Computer Vision, ICCV 2011 (2011)

2. Duygulu, P., Barnard, K., de Freitas, J.F.G., Forsyth, D.: Object Recognition as Machine Translation: Learning a Lexicon for a Fixed Image Vocabulary. In: Heyden, A., Sparr, G., Nielsen, M., Johansen, P. (eds.) ECCV 2002, Part IV. LNCS, vol. 2353, pp. 97-112. Springer, Heidelberg (2002)

3. Grubinger, M., Clough, P., Müller, H., Deselaers, T.: The iapr tc-12 benchmark - a new evaluation resource for visual information systems. In: Proc. of ICLRE (2006)

4. Farhadi, A., Endres, I., Hoiem, D., Forsyth, D.: Describing objects by their attributes. In: Proc. of IEEE International Conference on Computer Vision and Pattern Recognition, CVPR 2009 (2009)

5. Weston, J., Bengio, S., Usunier, N.: Large scale image annotation: learning to rank with joint word-image embeddings. Mach. Learn. 81, 21-35 (2010) 
6. Barnard, K., Duygulu, P., Forsyth, D., De Freitas, N., Blei, D.M., Jaz, K., Hofmann, T., Poggio, T., Shawe-taylor, J.: Matching words and pictures. Journal of Machine Learning Research 3, 1107-1135 (2003)

7. Yakhnenko, O., Honavar, V.: Annotating images and image objects using a hierarchical dirichlet process model. In: Proceedings of the 9th International Workshop on Multimedia Data Mining, MDM 2008: held in Conjunction with the ACM SIGKDD 2008, pp. 1-7. ACM, New York (2008)

8. Dalal, N., Triggs, B.: Histograms of oriented gradients for human detection. In: Proc. of IEEE International Conference on Computer Vision and Pattern Recognition (CVPR 2005), pp. 886-893 (2005)

9. Hertz, T., Bar-Hillel, A., Weinshall, D.: Learning distance functions for image retrieval. In: Proc. of IEEE International Conference on Computer Vision and Pattern Recognition (CVPR 2004), vol. 2, pp. II-570-II-577 (2004)

10. Guillaumin, M., Mensink, T., Verbeek, J., Schmid, C.: Tagprop: Discriminative metric learning in nearest neighbor models for image auto-annotation. In: Proc of IEEE International Conference on Computer Vision (ICCV 2009), pp. 309-316 (2009)

11. Makadia, A., Pavlovic, V., Kumar, S.: A New Baseline for Image Annotation. In: Forsyth, D., Torr, P., Zisserman, A. (eds.) ECCV 2008, Part III. LNCS, vol. 5304, pp. 316-329. Springer, Heidelberg (2008)

12. Wang, X.J., Zhang, L., Liu, M., Li, Y., Ma, W.Y.: Arista - image search to annotation on billions of web photos. In: Proc of IEEE International Conference on Computer Vision and Pattern Recognition (CVPR 2010), pp. 2987-2994 (2010)

13. Cesa-Bianchi, N., Gentile, C., Zaniboni, L.: Incremental algorithms for hierarchical classification. J. Mach. Learn. Res. 7, 31-54 (2006)

14. Bi, W., Kwok, J.: Multi-label classification on tree- and dag-structured hierarchies. In: Getoor, L., Scheffer, T. (eds.) Proceedings of the 28th International Conference on Machine Learning (ICML 2011), pp. 17-24. ACM, New York (2011)

15. Agrawal, R., Srikant, R.: Fast Algorithms for Mining Association Rules in Large Databases. In: VLDB 1994, Proceedings of 20th International Conference on Very Large Data Bases, pp. 487-499 (1994)

16. Gilbert, A., Bowden, R.: igroup: Weakly supervised image and video grouping. In: Proc. of International Conference on Computer Vision, ICCV 2011 (2011)

17. Lowe, D.: Distinctive Image Features from Scale-invariant Keypoints. Proc of International Jounral of Computer Vision (IJCV) 60, 91-110 (2004)

18. Cai, H., Mikolajczyk, K., Matas, J.: Learning linear discriminant projections for dimensionality reduction of image descriptors. IEEE Transactions on Pattern Analysis and Machine Intelligence (2010)

19. Agrawal, R., Imielinski, T., Swami, A.: Mining Association Rules between Sets of Items in Large Databases. In: Proc. of the 1993 ACM SIGMOD International Conference on Management of Data SIGMOD 1993, pp. 207-216 (1993)

20. Huiskes, M., Lew, M.: The mir flickr retreieval evaluation. In: Proc of MIR (2008)

21. Oliva, A., Torralba, A.: Modelling the shape of the scene: a holistic representation of the spatial envelope. Proc of International Journal of Computer Vision, IJCV $200142(3), 145-175$ (2001)

22. Nowak, S.: Overview of the Photo Annotation Task in ImageCLEF@ICPR. In: Ünay, D., Çataltepe, Z., Aksoy, S. (eds.) ICPR 2010. LNCS, vol. 6388, pp. 138-151. Springer, Heidelberg (2010) 